Published in final edited form as:

J Phys Chem B. 2019 June 13; 123(23): 4844-4849. doi:10.1021/acs.jpcb.9b01571.

\title{
Perturbation of Short Hydrogen Bonds in Photoactive Yellow Protein via Noncanonical Amino Acid Incorporation
}

\author{
Benjamin Thomson ${ }^{\dagger}$, Johan Both ${ }^{\ddagger}$, Yufan Wu§ ${ }^{\S}$ Robert M. Parrish, Todd J. Martínez, Steven \\ G. Boxer ${ }^{*}$ \\ Department of Chemistry, Stanford University, Stanford, California 94305, United States
}

\begin{abstract}
Photoactive yellow protein (PYP) is a small photoreceptor protein that has two unusually short hydrogen bonds between the deprotonated $p$-coumaric acid chromophore and two amino acids, a tyrosine and a glutamic acid. This has led to considerable debate as to whether the glutamic acidchromophore hydrogen bond is a low barrier hydrogen bond, with conflicting results in the literature. We have modified the $\mathrm{p} K_{\mathrm{a}}$ of the tyrosine by amber suppression and of the chromophore by chemical substitution. X-ray crystal structures of these modified proteins are nearly identical to the wild-type protein, so the heavy atom distance between proton donor and acceptor is maintained, even though these modifications change the relative proton affinity between donor and acceptor. Despite a considerable change in relative proton affinity, the NMR chemical shifts of the hydrogen-bonded protons are only moderately affected. QM/MM calculations were used to explore the protons' potential energy surface and connect the calculated proton position with empirically measured proton chemical shifts. The results are inconsistent with a low barrier hydrogen bond but in all cases are consistent with a localized proton, suggesting an ionic hydrogen bond rather than a low barrier hydrogen bond.
\end{abstract}

\section{Graphical Abstract}

\footnotetext{
*Corresponding Author Sboxer@stanford.edu.

$\dagger$ B.T.: Department of Chemistry and Chemical Biology, Harvard University, Cambridge, MA 02138.

‡J.B.: Boston Consulting Group, San Francisco, CA 94111.

§Y.W.: Department of Biological Chemistry and Molecular Pharmacology, Harvard Medical School, Boston, MA 02115.

Supporting Information

The Supporting Information is available free of charge on the ACS Publications website at DOI: 10.1021/acs.jpcb.9b01571.

Detailed experimental methods (S.1, S.2), X-ray crystallography data collection statistics (S.3), UV-vis spectroscopic data (S.4, S.5), protein NMR spectra (S.6), detailed computational methods (S.7), and H-bonding proton chemical shift predictions (S.8) (PDF) Optimized QM/MM structures (ZIP)

The authors declare no competing financial interest.
} 


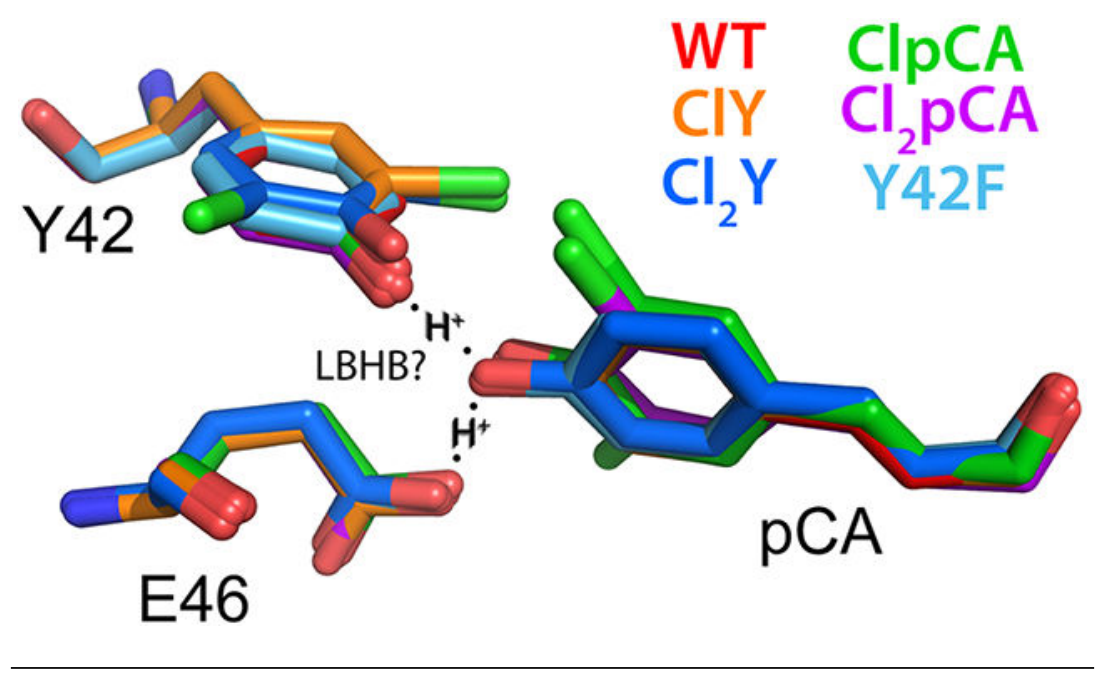

\section{INTRODUCTION}

Short hydrogen bonds, hydrogen bonds (H-bonds) with donor-acceptor (DA) distances less than $2.7 \AA$, are a common motif in enzyme active sites, ${ }^{1}$ and as such, their role in enzymatic catalysis is the subject of intense scrutiny and debate. ${ }^{2-4}$ This prevalence of short H-bonds has led to the suggestion that an active site H-bond whose DA distance is shorter than 2.5 Å could benefit energetically from being a so-called "low-barrier H-bond" (LBHB) ${ }^{2}$. LBHBs are a particular class of short H-bonds in which the proton's zero point energy exceeds the potential energy barrier to DA proton transfer (Figure 1A). LBHBs require not only short DA distances under $\sim 2.5 \AA$ but also very close matching of DA proton affinities. ${ }^{2}$ Under these conditions, the proton could be delocalized between the donor and acceptor, and LBHBs are predicted to be roughly 10 to $20 \mathrm{kcal}$ per mol stronger than normal H-bonds. ${ }^{5,6}$ For example, formation of a LBHB with a substrate's transition state (but not the reactant or product ground states) could facilitate reaction catalysis. ${ }^{7}$

There is, however, considerable debate over whether LBHBs are necessary to describe the transition state stabilization afforded by short H-bond formation, as well as whether LBHBs ever exist in protein environments. ${ }^{8-11}$ An alternative paradigm suggests that conventional electrostatic factors sufficiently explain the transition state stabilization provided by short $\mathrm{H}$ bonds and that short $\mathrm{H}$-bonds found in enzyme active sites are more likely to be short ionic H-bonds (SIHBs) due to polar solvation ${ }^{8}$ (Figure 1B). SIHBs are defined as ionic H-bonds that satisfy the length requirements of a LBHB yet contain a proton that is localized on one of the D or A heteroatoms. ${ }^{11-13}$ In contrast to LBHBs, SIHBs can occur between heteroatoms with mismatched proton affinities.

The proton in a LBHB is expected to exhibit a far downfield ${ }^{1} \mathrm{H}$ NMR chemical shift due to the deshielding caused by the proton not being closely associated with either H-bonding heteroatom. ${ }^{2,14,15}$ Thus, putative LBHBs in proteins are typically identified indirectly via their particularly short DA distances ${ }^{16}$ or by the unusually downfield ${ }^{1} \mathrm{H}$ NMR chemical shifts of their protons, ${ }^{17,18}$ however, DA distance and/or chemical shift information are not sufficient to conclude that a particular H-bond is a LBHB. The most direct methods for 
assessing LBHB presence are those that reveal the location of a H-bond's proton, such as neutron diffraction or ultrahigh resolution X-ray crystallography, ${ }^{13}$ noting that those methods report on the H-bond in a crystal environment rather than in solution. In at least one case, ultrahigh resolution X-ray crystallography has shown that an H-bond previously thought to be a LBHB due to its proton chemical shift ${ }^{17}$ is likely instead a SIHB. ${ }^{13}$

Structural investigations of the Halorhodospira halophila photoactive yellow protein (PYP) have identified two short H-bonds between the protein's deprotonated $p$-coumaric acid chromophore (pCA) and nearby amino acids Y42 and E46, with Y42-pCA and E46-pCA Hbond lengths (heavy atom separations) of 2.48 and $2.58 \AA$, respectively ${ }^{16,19}$ (Figure 2A). In 2009, Yamaguchi et al. published a $1.5 \AA$ A neutron diffraction crystal structure of PYP which appeared to show a deuteron centered in between the two oxygens in the E46-pCA H-bond. ${ }^{20}$ This data was interpreted by Yamaguchi and co-workers as the first direct evidence of a LBHB in a protein. Subsequently, Yamaguchi and others have invoked the proposed E46pCA LBHB to explain the behavior of PYP in solution, which is only reasonable if a LBHB exists in PYP in both crystals and in solution. ${ }^{21-23}$

The interpretation of Yamaguchi's neutron structure has been challenged by several computational studies. ${ }^{24-28} \mathrm{QM} / \mathrm{MM}$ calculations suggest that a LBHB between E46 and pCA could only possibly exist in particular circumstances: in the gas phase, ${ }^{27}$ if the Y42pCA H-bond were not present, ${ }^{26}$ or if the side chain of R52 (Figure 2A) were deprotonated, 24,28 an energetically improbable occurrence for a solvent-exposed arginine at neutral $\mathrm{pH}$.

Recent NMR experiments have shown that R52 is indeed protonated in solution, ${ }^{29}$ and while these NMR experiments do not indicate the protonation state of R52 in the crystal state, Groenhof et al. noted solvent pores in the PYP crystal lattice next to R52, implying that R52 is solvated by water and should be protonated in the $\mathrm{pH}$ range under investigation. ${ }^{27}$ Furthermore, Groenhof reanalyzed Yamaguchi's raw diffraction data and found that structures containing both a protonated R52 and a localized E46 proton are within the data's margins of error. ${ }^{27}$

It is also unclear whether E46 and deprotonated pCA are sufficiently close in proton affinity to support a LBHB. The solution $\mathrm{p} K_{\mathrm{a}}$ 's of pCA bound to the denatured PYP backbone (9.1) and free glutamic acid (4.25) are significantly different. It is well-known that the proton affinity of a residue can change significantly upon burial in a protein, and NMR titration experiments have shown little or no change in the protonation state of pCA in the folded protein between $\mathrm{pH} 3.4$ and 11.4, ${ }^{30}$ implying that the proton affinities of the chromophore and $\mathrm{E} 46$ in the folded protein environment are altered from those in solution. QM/MM calculated $\mathrm{p} K_{\mathrm{a}}$ 's of pCA and E46 in the protein environment, 5.4 and 8.6, respectively, ${ }^{24}$ agree with the localized proton placement produced by other QM/MM calculations.

On the basis of the available data, it is more reasonable to conclude that the E46-pCA interaction is a SIHB rather than a LBHB, but the LBHB hypothesis continues to be invoked to explain observations of the PYP active site. ${ }^{21-23,31}$ In the following we investigate the proposed LBHB by perturbing it using amber suppression and artificial chromophore incorporation, coupled to X-ray crystallography, ${ }^{1} \mathrm{H}$ NMR spectroscopy, and QM/MM computations. To examine the H-bond between E46 and the chromophore, we modified the 
hydrogen bond by producing five altered PYP variants with differentially perturbed E46chromophore differential acidities ( $\Delta \mathrm{p} K_{a}$ 's) (Figure 2B). We use the convention $\Delta \mathrm{p} K_{a}$ to describe the closeness of acidity matching required to form an internal LBHB to avoid confusion with $\mathrm{p} K_{\mathrm{a}}$ as defined in aqueous solution when accounting for the proton affinity difference between solvated and buried acidic residues. ${ }^{32,33}$ Since a LBHB requires a $\Delta \mathrm{p} K_{a}$ near zero, ${ }^{2,32}$ it is unlikely that more than one of these six PYPs contains a LBHB between $\mathrm{E} 46$ and the chromophore, due to varying $\Delta \mathrm{p} K_{a}$ between variants. Thus, if WT PYP contained a LBHB, such $\mathrm{p} K_{a}$ perturbation should result in destruction of the LBHB and marked changes in observable indicators of a LBHB presence such as the ${ }^{1} \mathrm{H}$ NMR chemical shift of the E46-pCA proton. Because LBHBs have strict geometric constraints, ${ }^{2}$ we ensured that the chromophore pocket geometry was conserved across all of our variants by X-ray crystallography. We also predicted the E46 proton one-dimensional DA potential energy surface and proton positional expectation value in each variant, using crystallographic coordinates to initialize our QM/MM computations. Finally, we used our computations in conjunction with an empirical relationship between proton position and ${ }^{1} \mathrm{H}$ NMR shift to generate predictions of E46 proton ${ }^{1} \mathrm{H}$ NMR shifts for each variant. We then compared experimental ${ }^{1} \mathrm{H}$ NMR shifts to our predicted shifts and to those predicted by a LBHB model.

\section{METHODS}

A full description of experimental methods and QM/MM methodology can be found in the supplementary text.

\section{EXPERIMENTAL RESULTS}

To significantly perturb the H-bond between the chromophore and E46, we increased $\Delta \mathrm{p} K_{a}$ by modifying the proton affinity of the chromophore both directly and indirectly. Direct perturbation was accomplished by incorporation of chlorinated chromophore analogues (Figure 2B), which changes the phenolate oxygen's proton affinity as seen by a change in its $\mathrm{p} K_{\mathrm{a}}$ under denaturing conditions (Figure $\mathrm{S} 4$ ). The solution $\mathrm{p} K_{\mathrm{a}}$ 's of the $\mathrm{pCA}$ phenolate oxygens were found to be 9.07, 7.56, and 5.74 for WT, ClpCA, and $\mathrm{Cl}_{2} \mathrm{pCA}$, respectively. Indirect perturbation was accomplished by exploiting the complementary nature of the chromophore's hydrogen bonding network ${ }^{26,34}$ and modifying the acidity of Y42 by substituting chlorinated tyrosine residues at position 42 (Figure 2B) using amber suppression, as well as the conventional mutation $\mathrm{Y} 42 \mathrm{~F}$. The solution $\mathrm{p} K_{\mathrm{a}}$ 's of the tyrosine phenolate oxygens have been shown to be 9.1, 8.3, and 6.4 for WT, ClY, and $\mathrm{Cl}_{2} \mathrm{Y}$, respectively. ${ }^{35}$ The absorption spectrum of the chromophore in these variants shifts systematically (Figure S3).

We determined the crystal structures of the variants $\mathrm{ClY}, \mathrm{Cl}_{2} \mathrm{Y}, \mathrm{ClpCA}$, and $\mathrm{Cl}_{2} \mathrm{pCA}$; structures for WT (PDB 1NWZ) and Y42F (PDB 1F9I) PYP have been published previously. ${ }^{19,36}$ The structures overlay very closely with WT as shown in Figure 2C; details of the structure determination and important DA distances are given in Tables S1 and S2, along with electron density maps in Figure S2. The presence of either a SIHB or LBHB is contingent on the general preservation of the chromophore H-bonding geometry, in 
particular the DA distance of the E46-chromophore H-bond. As shown in Table S3, we found that in each variant except $\mathrm{Cl}_{2} \mathrm{pCA}$, the E46-pCA DA distance required for a $\mathrm{LBHB}$ was conserved.

Proton NMR spectra were obtained for each variant in order to probe the chemical shift of the two protons between E46 and Y42 [Figure 3]. Both the Y42 and E46 H-bonding protons were detected in each variant, except Y42F in which the proton is absent. Our data for WT PYP matched what has been previously published. ${ }^{37} \mathrm{ClY}$ displayed four downfield peaks, rather than the expected two. This was interpreted as the presence of two coplanar solution conformations of the 3-chlorotyrosine residue with the ring flipped. Only one such conformation is observed in the crystallographic electron density map for ClY (Figure S2); however, that does not preclude two conformations from existing in solution-phase ClY. Multiple 3-chlorotyrosine conformations have been observed in the crystal structure of apoClY57 ketosteroid isomerase, a system with a similar short H-bonding network to ClY PYP. 38,39

There are only modest variations in the chemical shifts of the E46 proton among the conformationally similar variants (WT, ClY, Cl2Y, ClpCA, and Y42F). Discussion of peak assignments via NOESY data can be found in section S6.

\section{COMPUTATIONAL RESULTS AND DISCUSSION}

We used a combination of crystallographic coordinates, quantum mechanical geometry optimizations and scans, and empirical fits to predict ${ }^{1} \mathrm{H}$ NMR shifts from both the LBHB model and the SIHB model and compared those predicted shifts to measured values.

QM/MM geometry optimizations for each variant predicted a proton distance of approximately $1 \AA$ from the E46 oxygen, clearly localized on E46 (Figure 4). In conjunction with our experimental determination of the E46-chromophore DA distances for WT, ClY,

$\mathrm{Cl}_{2} \mathrm{Y}, \mathrm{ClpCA}$, and $\mathrm{Y} 42 \mathrm{~F}$, these predicted proton positions satisfy our definition of a SIHB for all of these variants. In the $\mathrm{QM} / \mathrm{MM}$ study, constrained optimizations (with the proton constrained to be transferred to the $\mathrm{pCA}$ oxygen atom) followed by relaxed optimizations were performed to attempt to find local minima with the proton transferred to the pCA oxygen atom. In all cases, when the constraint was removed, the proton relaxed back to the Y42 or E46 oxygen position. This indicates that in the absence of more-significant largescale conformational changes, all hydrogen bond potentials exhibit a single well, with the proton bound to the Y42 or E46 oxygen. We also investigated the effect of full QM treatment of the protein and its crystal water molecules. As shown in Figure S12 of the SI, the proton potential energy curve softens somewhat when the entire protein is treated quantum mechanically and more so when the fully QM protein is aqueously solvated with a polarizable continuum model. However, this softening is far from what would be required for a LBHB. The results from our QM/MM study were used in conjunction with an empirical model developed from previously published data relating the ${ }^{1} \mathrm{H}$ NMR shift of a H-bonded proton to the proton's distance to the acceptor heteroatom. 
Jeffrey and Yeon ${ }^{14}$ compiled a set of small molecule ionic crystal DA distances determined via neutron diffraction and high-resolution X-ray crystallography, along with the associated H-bonding proton's solid state ${ }^{1} \mathrm{H}$ NMR chemical shifts. Jeffrey and Yeon noted an empirical linear relationship between these two observables across a range of DA distances. We identified their data set for ionic H-bonds as most relevant to PYP due to the deprotonated state of the chromophore and obtained a least-squares fit to model this data as shown in Figure S13. The linear fit was then used to make two sets of predictions of the PYP variants' E46 proton chemical shifts: a set of shifts assuming the existence of a SIHB, and a set of shifts assuming the existence of a LBHB. The SIHB set was produced by using the linear fit in conjunction with proton positions predicted by our QM/MM study, while the LBHB set was produced by using the linear fit in conjunction with proton-acceptor distances derived by dividing our measured E46 DA distance in two, under the assumption that a LBHB would contain a perfectly centered proton. These predictions were plotted against the experimental E46 proton chemical shifts for each variant (Figure 5). It should be noted that, while we have included $\mathrm{Cl}_{2} \mathrm{pCA}$ in this analysis, we do not claim that it has a short enough DA distance to be a SIHB or LBHB. We instead classify it as a traditional ionic H-bond due to its longer DA distance. The range of DA distances covered by the linear fit produced from Jeffrey and Yeon's data includes that of $\mathrm{Cl}_{2} \mathrm{pCA}$, as well as the shorter DA distances of WT, $\mathrm{ClY}, \mathrm{Cl}_{2} \mathrm{Y}$, ClpCA, and $\mathrm{Y} 42 \mathrm{~F}$.

The experimental shifts (Figure 3) for WT, $\mathrm{ClY}, \mathrm{Cl}_{2} \mathrm{Y}, \mathrm{ClpCA}$, and $\mathrm{Y} 42 \mathrm{~F}$ were all within a $1 \sigma$ confidence interval of the QM/MM SIHB predicted shifts, while the experimental shift for $\mathrm{Cl}_{2} \mathrm{pCA}$ was slightly outside the $1 \sigma$ confidence interval of its QM/MM predicted shift. However, the experimental shifts for all variants were far outside the $1 \sigma$ confidence interval of the LBHB predicted shifts. Furthermore, there is little absolute difference in the E46 proton chemical shift between WT and the variants with conserved E46-chromophore DA bond geometry but assuredly altered $\Delta \mathrm{p} K_{a}$. All shifts in the group of variants WT, ClY, $\mathrm{Cl}_{2} \mathrm{Y}$, and ClpCA fall within the range of 14.6 to $15.6 \mathrm{ppm}$. Both this lack of significant change in the E46 proton chemical shift among variants of differing $\Delta \mathrm{p} K_{a}$ and the agreement of empirically predicted chemical shifts for $\mathrm{H}$-bonds with localized protons fail to support the hypothesis that a LBHB exists between E46 and the chromophore. A normal ionic hydrogen bond model is able to accurately explain the measured ${ }^{1} \mathrm{H}$ NMR shift for $\mathrm{Cl}_{2} \mathrm{pCA}$.

\section{CONCLUSIONS}

We investigated the existence of a proposed LBHB in $H$. halophila PYP by perturbing that $\mathrm{H}$-bond directly. Because LBHBs require close $\mathrm{p} K_{\alpha}$ matching between the DA partners, ${ }^{2,39}$ varying $\Delta \mathrm{p} K_{a}$ between the two DA partners in the E46-chromophore H-bond in a series of variants was expected to significantly alter the behavior of a LBHB, if present. At most one, if any, of these variants could reasonably be expected to contain a LBHB, which would be identifiable from QM/MM computations and NMR experiments. ${ }^{2,14,40}$ If one were not present, the behavior of this series of variants should be adequately explained by an alternative SIHB model. We created such a series of six PYP variants with varying $\Delta \mathrm{p} K_{a}$ by utilizing artificial chromophore incorporation, amber nonsense suppression, and 
conventional mutagenesis, but found no evidence to support the notion that a LBHB is present.

X-ray crystal structures were used to initiate QM/MM geometry optimizations of the E46 protons' optimized one-dimensional potential energy surfaces. These computations predicted that none of our variants contained a LBHB. Rather, they were all predicted to be SIHBs or conventional ionic H-bonds. We then tested these predictions experimentally through ${ }^{1} \mathrm{H}$ NMR using an established empirical linear relationship ${ }^{14}$ between the DA distance and ${ }^{1} \mathrm{H}$ NMR chemical shift of the proton in short H-bonds. Application of this linear fit showed, for every variant, good agreement between the variant's experimental E46 proton chemical shifts with the DA distances predicted by the QM/MM calculations. None of the variants showed agreement between experimental chemical shifts and chemical shifts expected from LBHB protons. Furthermore, the E46 proton chemical shifts of the WT, ClY, $\mathrm{Cl}_{2} \mathrm{Y}$, and ClpCA variants were all relatively similar to one another $( \pm 0.6 \mathrm{ppm})$, indicating similar degrees of shielding for the protons in all of these variants and contradicting the notion that one of these variants has a fundamentally different proton environment. These experimental observations agree with our QM/MM predictions and do not support the presence of a LBHB in any of the PYP variants.

\title{
Supplementary Material
}

Refer to Web version on PubMed Central for supplementary material.

\section{ACKNOWLEDGMENTS}

\begin{abstract}
We thank Professor Jiangyun Wang for the gift of 3-chlorotyrosine and 3,5-dichlorotyrosine nonsense suppression systems and Dr. Marc Deller and the Stanford Macromolecular Structure Knowledge Center, as well as staff at SSRL and ALS, for expertise in protein crystallization and X-ray data collection. We also acknowledge Dr. Jeffrey Pelton and Dr. Stephen Lynch of the Berkeley QB3 and Stanford Chemistry NMR facilities, respectively, for their assistance and expertise on collecting NMR data. Part of this work was supported by NIH R35 GM118044 (to S.G.B.); support from the National Science Foundation's CCI Phase I: NSF Center for First-Principles Design of Quantum Processes (CHE-1740645) is gratefully acknowledged.
\end{abstract}

\section{REFERENCES}

(1). Rajagopal S; Vishveshwara S Short Hydrogen Bonds in Proteins. FEBS J. 2005, 272, 1819-1832. [PubMed: 15819878]

(2). Cleland WW; Frey PA; Gerlt JA The Low Barrier Hydrogen Bond in Enzymatic Catalysis. J. Biol. Chem 1998, 273, 25529-25532. [PubMed: 9748211]

(3). Katz BA; Spencer JR; Elrod K; Luong C; Mackman RL; Rice M; Sprengeler PA; Allen D; Janc J Contribution of Multicentered Short Hydrogen Bond Arrays to Potency of Active Site-Directed Serine Protease Inhibitors. J. Am. Chem. Soc 2002, 124, 11657-11668. [PubMed: 12296731]

(4). Jang DS; Choi G; Cha HJ; Shin S; Hong BH; Lee HJ; Lee HC; Choi KY Contribution of a LowBarrier Hydrogen Bond to Catalysis is Not Significant in Ketosteroid Isomerase. Mol. Cells 2015, 38, 409-415. [PubMed: 25947291]

(5). Garcia-Viloca M; Gonzalez-Lafont A; Lluch JM Theoretical Study of the Low-Barrier Hydrogen Bond in the Hydrogen Maleate Anion in the Gas Phase. Comparison with Normal Hydrogen Bonds. J. Am. Chem. Soc 1997, 119, 1081-1086.

(6). McAllister MA Characterization of Low-Barrier Hydrogen Bonds. 3. Hydrogen Maleate. An ab initio and DFT Investigation. Can. J. Chem 1997, 75, 1195-1202. 
(7). Cleland W; Kreevoy M Low-Barrier Hydrogen Bonds and Enzymic Catalysis. Science 1994, 264, 1887-1890. [PubMed: 8009219]

(8). Warshel A; Papazyan A; Kollman P On Low Barrier Hydrogen Bonds and Enzyme Catalysis. Science 1995, 269, 102-106. [PubMed: 7661987]

(9). Schutz CN; Warshel A The Low-Barrier Hydrogen Bond (LBHB) Proposal Revisited: The Case of the Asp-His Pair in Serine Protease. Proteins: Struct., Funct., Genet 2004, 55, 711-723. [PubMed: 15103633]

(10). Warshel A; Papazyan A Energy Considerations Show that Low-Barrier Hydrogen Bonds Do Not Offer a Catalytic Advantage over Ordinary Hydrogen Bonds. Proc. Natl. Acad. Sci. U. S. A 1996, 93, 13665-13670. [PubMed: 8942991]

(11). Kaledhonkar S; Hara M; Stalcup TP; Xie A; Hoff WD Strong Ionic Hydrogen Bonding Causes a Spectral Isotope Effect in Photoactive Yellow Protein. Biophys. J 2013, 105, 2577-2585. [PubMed: 24314088]

(12). Meot-Ner M The Ionic Hydrogen Bond. Chem. Rev 2005, 105, 213-284. [PubMed: 15729772]

(13). Fuhrmann C; Daugherty M; Agard D Subangstom Crystallography Reveals that Short Ionic Hydrogen Bonds, and Not a His-Asp Low-Barrier Hydrogen Bond, Stabilize the Transition State in Serine Protease Catalysis. J. Am. Chem. Soc 2006, 128, 9086-9102. [PubMed: 16834383]

(14). Jeffrey GA; Yeon Y The Correlation Between Hydrogen-Bond Lengths and Chemical Shifts in Crystals. Acta Crystallogr., Sect. B: Struct. Sci 1986, 42, 410-413.

(15). Pinney MM; Natarajan A; Yabukarski F; Sanchez DM; Doukov T; Schwans JP; Martinez TJ; Herschlag D; Liu F; Liang R Structural Coupling Throughout the Active Site Hydrogen Bond Networks of Ketosteroid Isomerase and Photoactive Yellow Protein. J. Am. Chem. Soc 2018, 140, 9827-9843. [PubMed: 29990421]

(16). Anderson S; Crosson S; Moffat K Short Hydrogen Bonds in Photoactive Yellow Protein. Acta Crystallogr., Sect. D: Biol. Crystallogr 2004, 60, 1008-1016. [PubMed: 15159559]

(17). Frey PA; Whitt SA; Tobin JB A Low-Barrier Hydrogen Bond in the Catalytic Triad of Serine Proteases. Science 1994, 264, 1927-1930. [PubMed: 7661899]

(18). Zhao Q; Abeygunawardana C; Talalay P; Mildvan A NMR Evidence for the Participation ofa Low-Barrier Hydrogen Bond in the Mechanism of $\Delta^{5}-3$-Ketosteroid Isomerase. Proc. Natl. Acad. Sci. U. S. A 1996, 93, 8220-8224. [PubMed: 8710850]

(19). Getzoff E; Gutwin K; Genick U Anticipatory Active-Site Motions and Chromophore Distortion Prime Photoreceptor PYP for Light Activation. Nat. Struct. Mol. Biol 2003, 10, 663-668.

(20). Yamaguchi S; Kamikubo H; Kurihara K; Kuroki R; Niimura N; Shimizu N; Yamazaki Y; Kataoka M Low-Barrier Hydrogen Bond in Photoactive Yellow Protein. Proc. Natl. Acad. Sci. U. S. A 2009, 106, 440-444. [PubMed: 19122140]

(21). Mizuno M; Kamikubo H; Kataoka M; Mizutani Y Changes in the Hydrogen-Bond Network around the Chromophore of Photoactive Yellow Protein in the Ground and Excited States. J. Phys. Chem. B 2011, 115, 9306-9310. [PubMed: 21688774]

(22). Kita Y; Kamikubo H; Kataoka M; Tachikawa M Theoretical Analysis of the Geometrical Isotope Effect on the Hydrogen Bonds in Photoactive Yellow Protein With Multi-Component Density Functional Theory. Chem. Phys 2013, 419, 50-53.

(23). Kanematsu Y; Kamikubo H; Kataoka M; Tachikawa M Vibrational Analysis on the Revised Potential Energy Curve of the Low-Barrier Hydrogen Bond in Photoactive Yellow Protein. Comput. Struct. Biotechnol. J 2016, 14, 16-19. [PubMed: 27274362]

(24). Saito K; Ishikita H Energetics of Short Hydrogen Bonds in Photoactive Yellow Protein. Proc. Natl. Acad. Sci. U. S. A 2012, 109, 167-172. [PubMed: 22173632]

(25). Saito K; Ishikita HH Atom Positions and Nuclear Magnetic Resonance Chemical Shifts of Short H Bonds in Photoactive Yellow Protein. Biochemistry 2012, 51, 1171-1177. [PubMed: 22263543]

(26). Saito K; Ishikita H Formation of an Unusually Short Hydrogen Bond in Photoactive Yellow Protein. Biochim. Biophys. Acta, Bioenerg 2013, 1827, 387-394.

(27). Graen T; Inhester L; Clemens M; Grubmüller H; Groenhof G The Low Barrier Hydrogen Bond in the Photoactive Yellow Protein: A Vacuum Artifact Absent in the Crystal and Solution. J. Am. Chem. Soc 2016, 138, 16620-16631. [PubMed: 27966904] 
(28). Nadal-Ferret M; Gelabert R; Moreno M; Lluch JM Are There Really Low-Barrier Hydrogen Bonds in Proteins? The Case of Photoactive Yellow Protein. J. Am. Chem. Soc 2014, 136, 3542 3552. [PubMed: 24548066]

(29). Yoshimura Y; Oktaviani NA; Yonezawa K; Kamikubo H; Mulder FA; Unambiguous Determination of Protein Arginine Ionization States in Solution by NMR Spectroscopy. Angew. Chem., Int. Ed 2017, 56, 239-242.

(30). Oktaviani NA; Pool TJ; Yoshimura Y; Kamikubo H; Scheek RM; Kataoka M; Mulder FAA Active-Site $\mathrm{pKa}$ Determination for Photoactive Yellow Protein Rationalizes Slow Ground-State Recovery. Biophys. J 2017, 112, 2109-2116. [PubMed: 28538148]

(31). Kuramochi H; Takeuchi S; Yonezawa K; Kamikubo H; Kataoka M; Tahara T Probing the Early Stages of Photoreception in Photoactive Yellow Protein with Ultrafast Time-Domain Raman Spectroscopy. Nat. Chem 2017, 9, 660-666. [PubMed: 28644485]

(32). Oltrogge LM; Boxer SG Short Hydrogen Bonds and Proton Delocalization in Green Fluorescent Protein. ACS Cent. Sci 2015, 1, 148-156. [PubMed: 27162964]

(33). Fried SD; Boxer SG Thermodynamic Framework for Identifying Free Energy Inventories of Enzyme Catalytic Cycles. Proc. Natl. Acad. Sci. U. S. A 2013, 110, 12271-12276. [PubMed: 23840058]

(34). Joshi CP; Otto H; Hoersch D; Meyer TE; Cusanovich MA; Heyn MP Strong Hydrogen Bond Between Glutamic Acid 46 and Chromophore Leads to the Intermediate Spectral Form and Excited State Proton Transfer in the Y42F Mutant of the Photoreceptor Photoactive Yellow Protein. Biochemistry 2009, 48, 9980-9993. [PubMed: 19764818]

(35). Liu X; Jiang L; Li J; Wang L; Yu Y; Zhou Q; Lv X; Gong W; Lu Y; Wang J Significant Expansion of Fluorescent Protein Sensing Ability through the Genetic Incorporation of Superior Photo-Induced Electron-Transfer Quenchers. J. Am. Chem. Soc 2014, 136, 13094-13097. [PubMed: 25197956]

(36). Brudler R; Meyer TE; Genick UK; Devanathan S; Woo TT; Millar DP; Gerwert K; Cusanovich MA; Tollin G; Getzoff ED Coupling of Hydrogen Bonding to Chromophore Conformation and Function in Photoactive Yellow Protein. Biochemistry 2000, 39, 13478-13486. [PubMed: 11063584]

(37). Sigala PA; Tsuchida MA; Herschlag D Hydrogen Bond Dynamics in the Active Site of Photoactive Yellow Protein. Proc. Natl. Acad. Sci. U. S. A 2009, 106, 9232-9237. [PubMed: 19470452]

(38). Wu Y; Fried SD; Boxer SG A Critical Test of the Electrostatic Contribution to Catalysis with Noncanonical Amino Acids in Ketosteroid Isomerase. Biochemistry 2015, 54, 7110-7119. [PubMed: 26571340]

(39). Wu Y; Fried SD; Boxer SG Dissecting Proton Delocalization in an Enzyme's Hydrogen Bond Network with Unnatural Amino Acids. Biochemistry 2015, 54, 7110-7119. [PubMed: 26571340]

(40). Hibbert F; Emsley J Hydrogen Bonding and Chemical Reactivity In Advances in Physical Organic Chemistry; Elsevier, 1990; Vol. 26, pp 255-379. 



Figure 1.

Characteristic 1D ${ }^{1} \mathrm{H}$ proton potential energy surfaces for (A) a short, $\mathrm{p} K_{a}$ matched LBHB and (B) a short, ionic hydrogen bond. The LBHB's barrier for proton transfer is lower than the proton zero-point energy (ZPE), leading to a delocalized proton with a positional expectation value exactly in between the two hydrogen bonding heteroatoms. 
A)

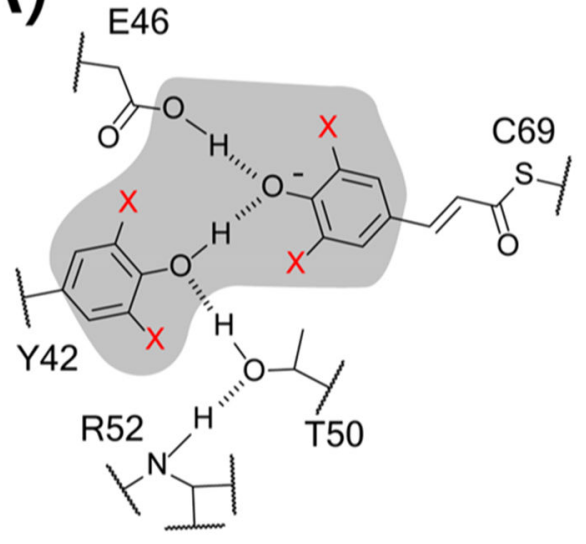

B)

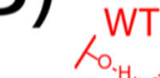<smiles>c1ccc(Oc2ccccc2)cc1</smiles><smiles>[AlH2]</smiles>

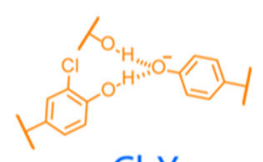

$\mathrm{Cl}_{2} \mathrm{Y}$

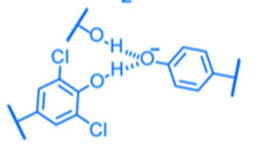

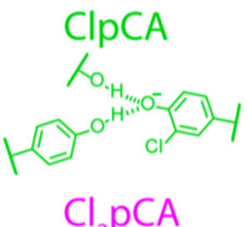

${ }_{2} \mathrm{pCA}$

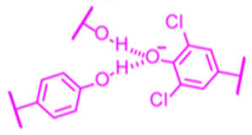

Y42F

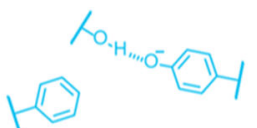

C)
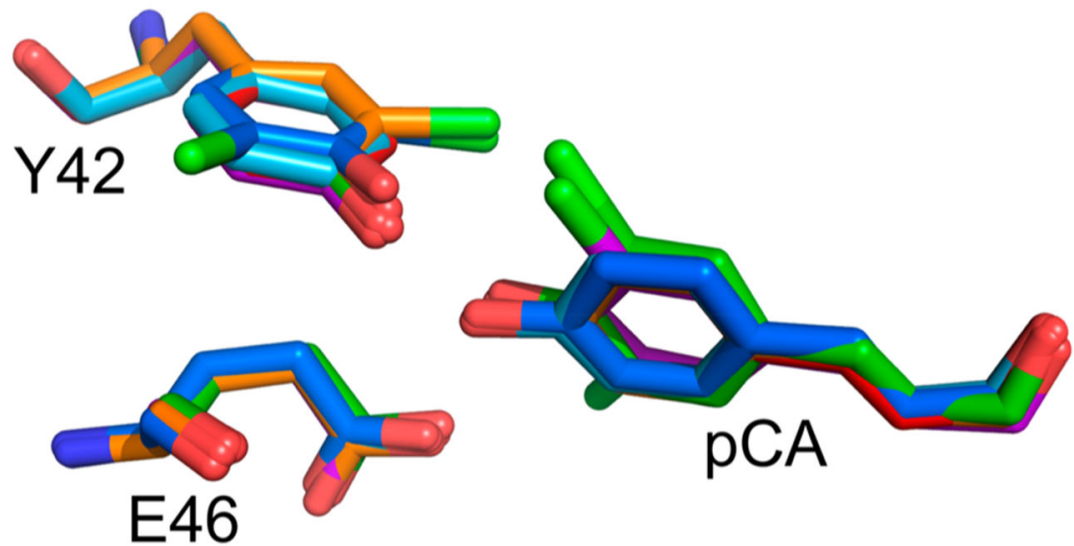

Figure 2.

(A) PYP chromophore and surrounding amino acids showing hydrogen bonding network, including the E46-chromophore hydrogen bond, suggested to be a LBHB. The region where changes have been made is highlighted in gray. Red X's show the locations of chlorosubstituents. (B) Variants and mutant studied here (color code used throughout). (C) Overlaid PYP variant active site X-ray structures (see Figure S2 for individual structures). 


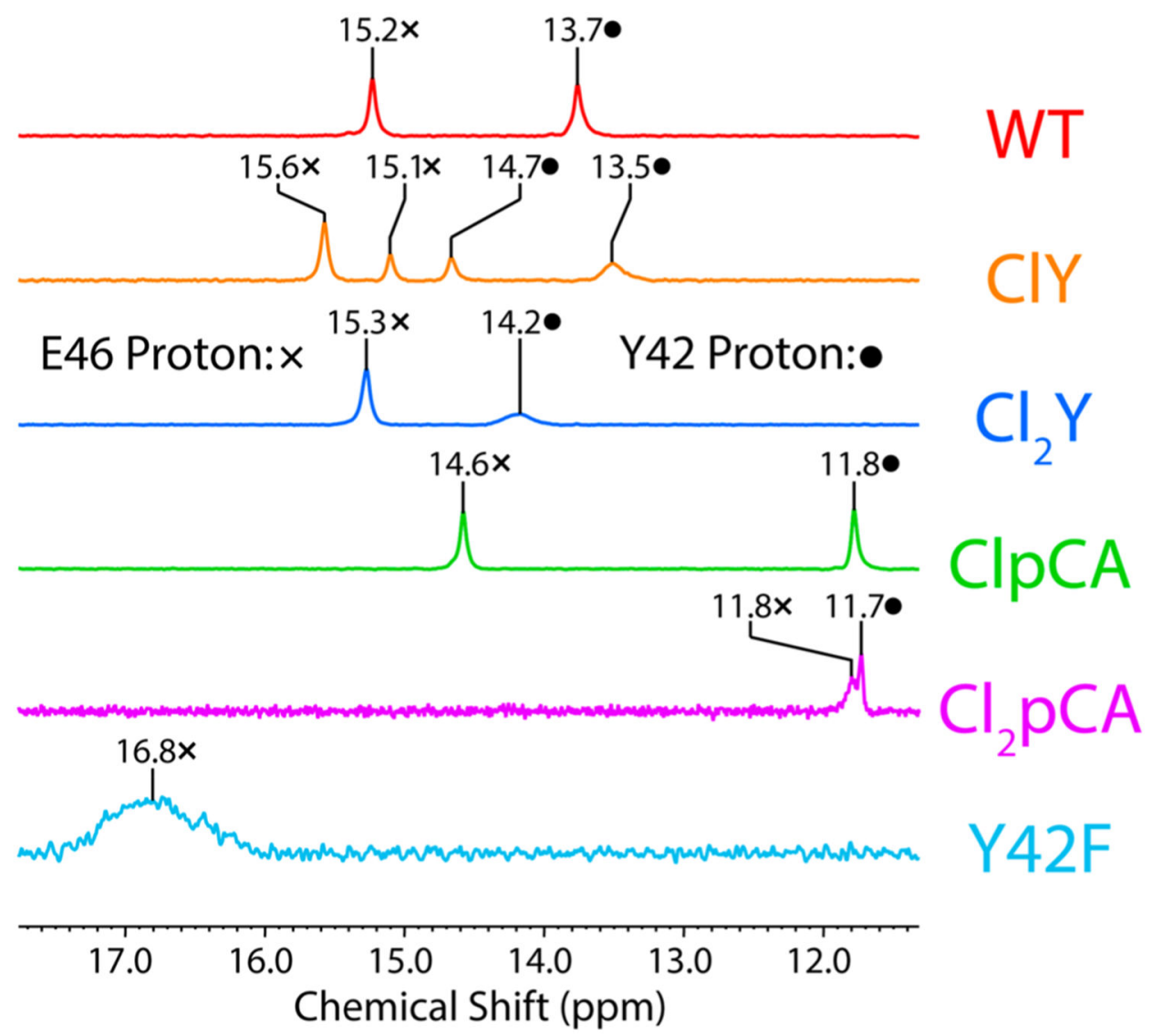

Figure 3.

Downfield 1D ${ }^{1} \mathrm{H}$ NMR spectra of each PYP variant. Peaks assigned to the E46 H-bonded proton are labeled with $\times$, while peaks assigned to the Y42 protons are labeled with (see Figures S5-S9 for details of assignment). The four peaks in the ClY spectrum are likely due to two ring flipped conformations of the 3 -chlorotyrosine residue observed in solution but not in the X-ray structures. 


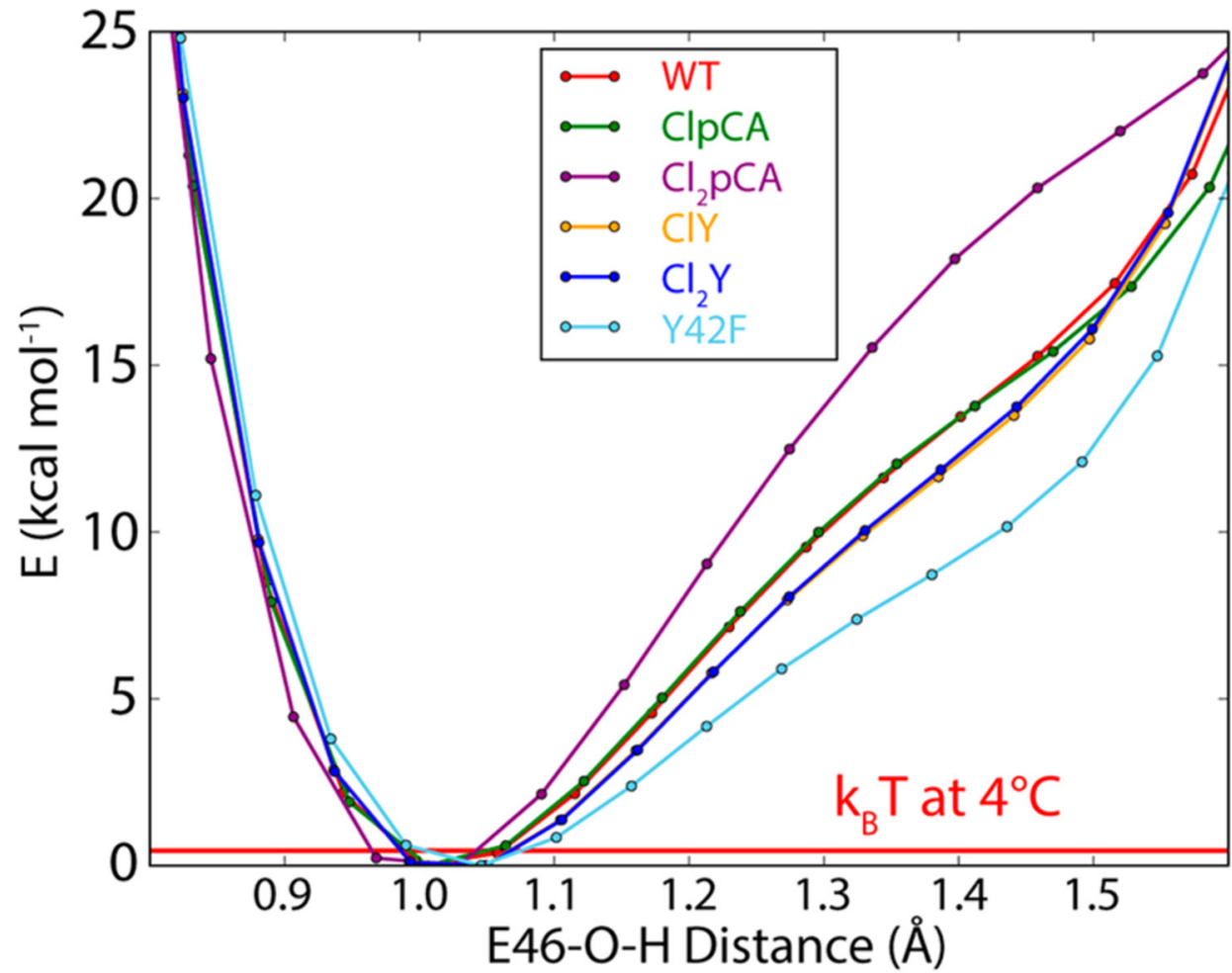

Figure 4.

QM/MM 1D potential energy surfaces (rigid proton scans) of proton transfer between E46 and the pCA chromophore, with scan performed from the QM/MM optimal geometry.

Computations were carried out at $\omega \operatorname{PBE}(\omega=0.3)-\mathrm{D} 3 / 6-31 \mathrm{G}^{* *} / / \mathrm{AMBER}-\mathrm{ff} 14 \mathrm{SB}$. Note the lack of a second potential energy minimum in any of the six variants and the predicted proton position at $4{ }^{\circ} \mathrm{C}$ approximately $1 \AA$ from the E46 oxygen in all variants. 


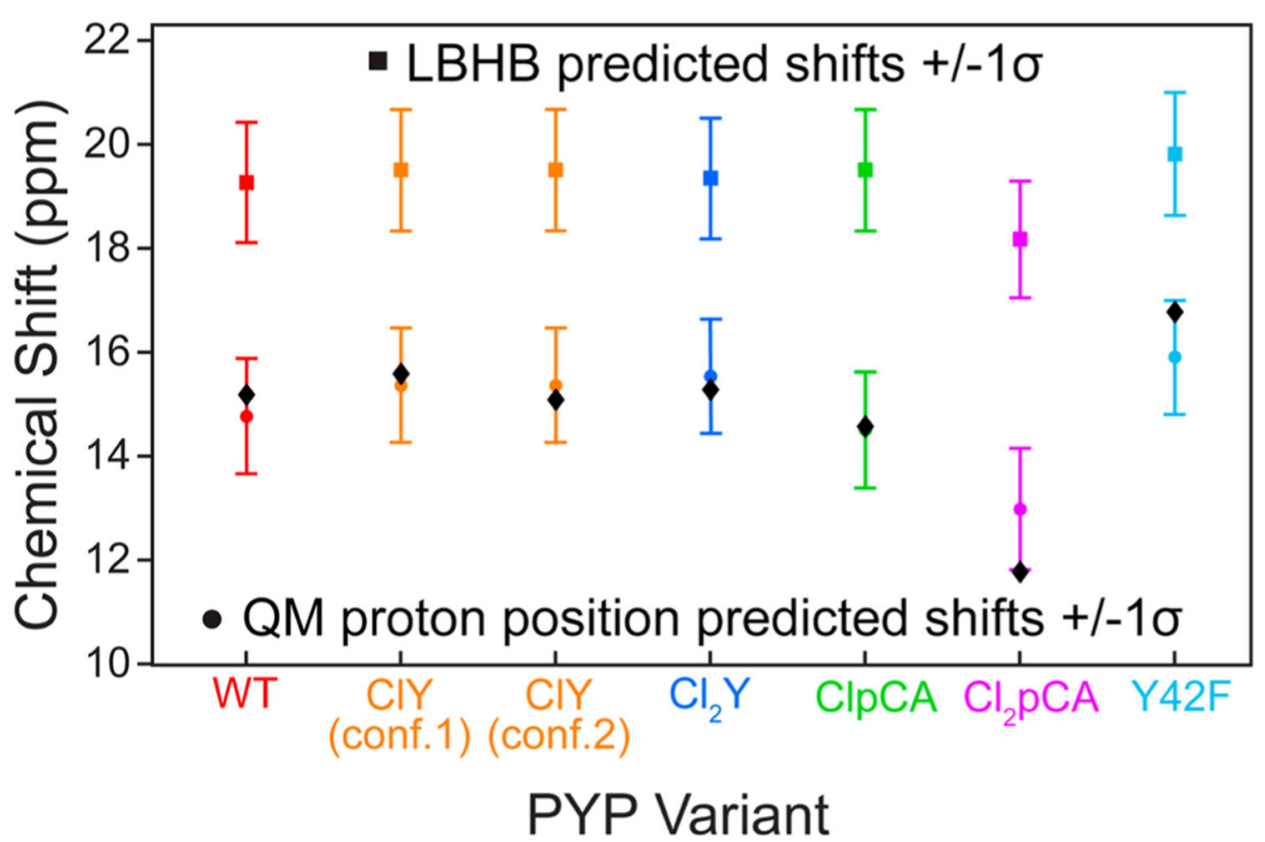

Figure 5.

Experimental E46 proton ${ }^{1} \mathrm{H}$ NMR chemical shifts for each variant $(\diamond)$, compared with corresponding empirical predictions of NMR chemical shift assuming the central proton position produced by a LBHB (square upper symbols), as well as assuming a SIHB (circle lower symbols) using the proton positions given by QM/MM computed predictions. Error bars represent a $1 \sigma$ deviation given by the linear fit statistics. The experimental data fit with the QM/MM predicted shift rather than with the LBHB predicted shift in each variant, as well as with the relatively small variance in shift between multiple variants despite varying $\Delta \mathrm{p} K_{a} . \mathrm{ClY}$ is listed twice, as we believe the 3-chlorotyrosine residue exists in two possible conformations in solution, leading to two different E46 ${ }^{1} \mathrm{H}$ NMR chemical shifts (see Figure 3 and text). 